\title{
Roll Quench System in Continuous Annealing Line*
}

\section{Introduction}

The continuous annealing process with water quenching system, known as NKK-CAL WQ Process, has already been working in the production of drawing quality cold rolled steel sheets and high strength cold rolled sheets. However, with the recent tendency toward energysaving, development of new rapid cooling processes has become necessary for further reduction of production cost. The Roll Quench (RQ) process meets this need. One optimum application of the RQ process is to combine the technology, capable of producing drawing quality cold rolled sheets at lower cost with the WQ process, capable of producing a wide range of products.

\section{Facility Specifications}

The line specifications and line arrangement are shown in Table 1 and Fig. 1, respectively. To obtain the required high cooling rate, 1 ) cooling roll materials, 2) the cooling roll shell thickness, 3) the cooling roll surface roughness, 4) the cooling roll interior water flow channels, and 5) contact pressure were thoroughly studied.

The strip temperature is controlled by adjusting the contact length between the strip and the cooling roll, and the cooling water flow rate.

Favorable strip threading conditions and uniform cooling are secured by a strip tension control mechanism, a cooling roll level control mechanism, and the proper crowning of the cooling rolls.

\section{Result}

(1) Strip cooling rates of between 150 and $450{ }^{\circ} \mathrm{C} / \mathrm{s}$ and heat transfer coefficients between 1500 and $2200 \mathrm{kcal} / \mathrm{m}^{2} \mathrm{~h}^{\circ} \mathrm{C}$ are obtained. (Fig. 2)

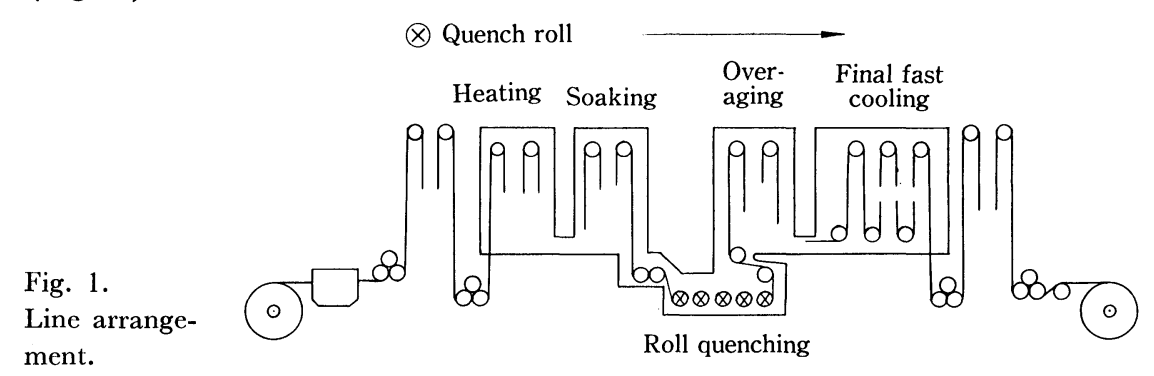

(2) The cooling end temperature is controllable within $\pm 5^{\circ} \mathrm{C}$.

(3) No reheating energy is required.

(4) The surface quality and shape of the products are excellent, and the mechanical properties are comparable with those of water quenched materials. Examples of properties are shown in Table 2.

\section{Conclusion}

The newly established RQ process is being used in combination with the water quenching process in cold rolled sheets manufacture to rationalize the line operation. The RQ technology also allows the use of coolants other than water to cool the rolls from the inside.

\begin{tabular}{|c|c|c|c|}
\hline \multirow{2}{*}{$\begin{array}{l}\text { Line } \\
\text { specification }\end{array}$} & Strip size & $\begin{array}{l}\text { Thickness } \\
\text { Width }\end{array}$ & $\begin{array}{l}0.4 \sim 1.6 \mathrm{~mm} \\
610 \sim 1300 \mathrm{~mm}\end{array}$ \\
\hline & \multicolumn{2}{|c|}{ Line speed } & $180 \mathrm{mpm} \max$. \\
\hline $\begin{array}{l}\text { Cooling roll } \\
\text { specification }\end{array}$ & $\begin{array}{l}\text { No. of rol } \\
\text { Roll mate } \\
\text { Contact } 1 \\
\text { Water flo }\end{array}$ & h & $\begin{array}{l}5 \\
\text { Copper (3) Steel (2) } \\
1.0 \sim 5.2 \mathrm{~m} \\
3.5 \sim 20 \mathrm{t} / \mathrm{hr} / \text { roll }\end{array}$ \\
\hline
\end{tabular}

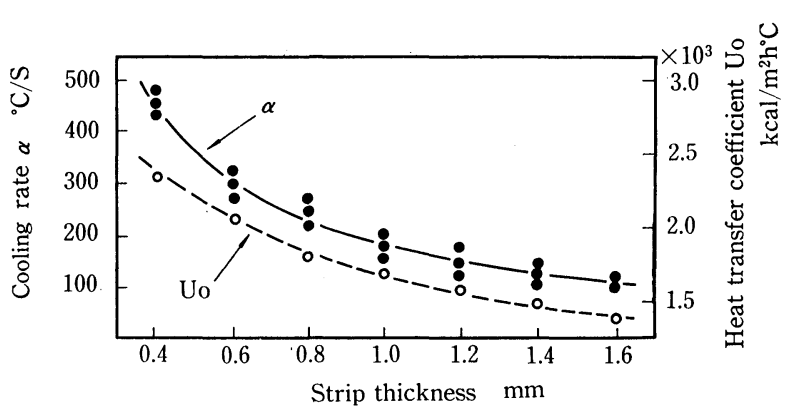

Fig. 2. RQ cooling characteristics curves.

Table 2. Manufacturing conditions and typical mechanical properties of RQ materials.

$*: \quad \mathrm{BH}$

\begin{tabular}{|c|c|c|c|c|c|c|c|c|c|c|c|c|c|c|c|c|c|c|}
\hline \multirow{2}{*}{ 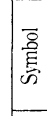 } & \multirow{2}{*}{ Steel type } & \multicolumn{7}{|c|}{ Chemical composition } & \multirow{2}{*}{$\begin{array}{l}\text { Cold } \\
\text { reduc- } \\
\text { tion } \\
(\%) \\
\end{array}$} & \multirow{2}{*}{$\begin{array}{l}\text { Heat } \\
\text { cycle }\end{array}$} & \multirow{2}{*}{$\begin{array}{l}\text { Thick. } \\
\text { ness } \\
\text { (mm) }\end{array}$} & \multicolumn{4}{|c|}{ Mechanical properties } & \multicolumn{2}{|c|}{ (JIS No.5) } & \multirow{8}{*}{ 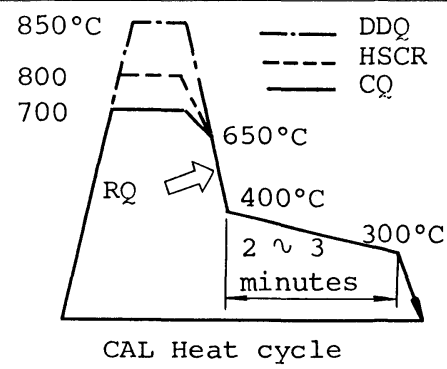 } \\
\hline & & c & $\mathrm{Si}$ & $\mathrm{Mn}$ & $\mathrm{P}$ & $\mathrm{s}$ & Sol.Al & $\mathrm{N}$ & & & & YP & YPEI & TS & $\mathrm{El}$ & $\mathrm{r}$ & A.I. & \\
\hline $\mathrm{A}$ & Capped steel & 0.047 & tr. & 0.32 & 0.013 & 0.014 & - & 0.0018 & 68 & $\mathrm{CQ}$ & 1.0 & 20.8 & 0 & 33.2 & 44.8 & 1.34 & 5.2 & \\
\hline B & Al-killed steel & 0.032 & 0.01 & 0.22 & 0.015 & 0.015 & 0.042 & & 68 & $\mathrm{CQ}$ & 1.0 & 20.7 & 0 & 33.5 & 44.5 & 1.40 & 3.6 & \\
\hline C & $\downarrow$ & $\downarrow$ & $\downarrow$ & $\downarrow$ & $\downarrow$ & $\downarrow$ & $\downarrow$ & $\downarrow$ & 68 & $\mathrm{DDQ}$ & 1.0 & 18.5 & 0 & 32.0 & 46.8 & 1.65 & 4.2 & \\
\hline $\mathrm{D}$ & $\downarrow$ & 0.017 & 0.01 & 0.14 & 0.008 & 0.016 & 0.036 & 0.0026 & 68 & $\mathrm{CQ}$ & 1.0 & 20.2 & 0 & 32.2 & 46.8 & 1.46 & 4.6 & \\
\hline $\mathrm{E}$ & $\downarrow$ & $\downarrow$ & $\downarrow$ & $\downarrow$ & $i$ & $\downarrow$ & $\downarrow$ & $\downarrow$ & 68 & DDQ & 1.0 & 18.0 & 0 & 31.0 & 47.8 & 1.70 & 4.8 & \\
\hline $\mathrm{F}$ & $\downarrow$ & 0.020 & 0.23 & 0.17 & 0.030 & 0.004 & 0.032 & 0.0010 & 80 & $\mathrm{HSCR}$ & 0.75 & 24.0 & 0 & 36.6 & 41.0 & 1.72 & *4.5 & \\
\hline
\end{tabular}

* For further information, write to Technical Department for Steel Products, Planning \& Development, Nippon Kokan K.K., 1-1-2, Marunouchi, Chiyoda-ku, Tokyo 100. 